\title{
Spatial, Temporal, and Spatiotemporal Variation of Malaria Incidence and Risk Factors in West Gojjam Zone From 1 July 2013- 30 June 2018, Northwest Ethiopia, 2019.
}

Eniyew Tegegne Bayih ( $\sim$ eniyewtegegne@gmail.com )

Debre Markos University College of Health Science https://orcid.org/0000-0002-1915-2229

Kassahun Alemu Gelaye

University of Gondar

Awrajaw Dessie Zeleke

University of Gondar

Alebachew Shimelash Damtew

Debre Markos University

Biachew Asmare Asmare

Debre Markos University

Yikeber Argachew Demil

Debre Markos University

Yonas Lamore Lamore

Debre Markos University

Tegegne Temesgen Temesgen

Debre Markos University

Biruk Demissie Melessie

Debre Tabor University

\section{Research}

Keywords: Spatiotemporal Variation, Malaria Incidence, Risk Factor, West Gojjam, Ethiopia

Posted Date: March 11th, 2020

DOI: https://doi.org/10.21203/rs.3.rs-16733/v1

License: (c) (i) This work is licensed under a Creative Commons Attribution 4.0 International License. Read Full License 


\section{Abstract}

Background: Malaria is a life-threatening acute febrile illness which is affecting the lives of millions globally. Its distribution is characterized by spatial, temporal, and spatiotemporal heterogeneity making detection of the spacetime distribution and mapping high-risk areas useful to effectively targeting hot spots of malaria for intervention. Methods : Time series cross sectional study was conducted using data obtained from weekly malaria surveillance reports stored in the Amhara Public Health Institute from 1 July 2013-30 June 2018. Climatic variables were obtained from West Amhara Meteorological Agency. All districts were included and geo-coded and the spatial data was created in ArcGIS10.2.2 software. Global and local spatial autocorrelation were used to test the hypothesis and to detect hot spots respectively. The Poisson model was fitted to determine the purely spatial, temporal, and space-time clusters using SaTScan ${ }^{\mathrm{Tm}} 9.6$ software. Spearman correlation, bivariate, and multivariable negative binomial regressions were used to analyze the relation of the climatic factors to count of malaria incidence. Result: The study revealed spatial, temporal, and spatiotemporal heterogeneity of malaria incidence. Jabitenan, Quarit, Sekela, Bure, and Wonberma were high rate spatial cluster of malaria incidence hierarchically. Spatiotemporal clusters were detected. A temporal scan statistic identified one risk period from 1 July 2013 to 30 June 2015. Monthly average temperature was positively but monthly average rainfall and monthly average relative humidity were negatively correlated to count of malaria incidence at all lag-months. The adjusted incidence rate ratio showed that monthly average temperature and monthly average rainfall were independent predictors for malaria incidence at all lag-months. Monthly average relative humidity was significant at 2 months lag. Conclusion: Malaria incidence had shown spatial, temporal, spatiotemporal variability in West Gojjam zone. Mean monthly temperature and rainfall were directly and inversely associated to count of malaria incidence respectively. Considering these space-time variations and risk factors (temperature and rainfall) would be useful for the prevention and control and ultimately achieve elimination. Keywords: Spatiotemporal Variation, Malaria Incidence, Risk Factor, West Gojjam, Ethiopia

\section{Background}

Malaria is a common and life-threatening acute febrile illness in many tropical and subtropical areas caused by five different parasites of protozoan species: $P$. falciparum, $P$. malariae, P. ovale $P$. vivax, and P. knowlesi. It is transmitted by female Anopheles mosquitoes, which bite mainly between dusk and dawn(1). P. falciparum is a fatal form if treatment is delayed (2). Other species result in significant morbidity but are rarely life-threatening (3-6).

The vision of the World Health Organization (WHO) is a world free of malaria by ensuring universal access to malaria prevention, diagnosis, and treatment, accelerating efforts towards elimination, transforming malaria surveillance, harnessing innovation and expanding research and strengthening the enabling environment. Despite interventions, gains achieved are fragile and unevenly distributed. The human toll of malaria remains high due to lack of robust, predictable and sustained financing compounded by the difficulty in maintaining political commitment and ensuring regional collaboration. Since 2014, investments in malaria control have declined in many high-burden countries. The second important challenge is biological: the emergence of parasite resistance to anti-malarial medicines and of mosquito resistance to insecticides $(4,7)$.

Globally the burden of malaria in 2016 alone was a total of 216 million cases, an increase of 5 million cases over the previous year (2015) which was about 211 million. Although malaria case incidence has fallen globally since 2010, the rate of decline has stalled and even reversed in some regions since 2014. Mortality rates have followed a similar pattern(4). 
The WHO African Region accounts for about $90 \%$ of malaria cases and deaths worldwide where one child in 10 dies before the age of 5 from malaria. Fifteen countries - all but one in sub-Saharan Africa - carry $80 \%$ of the global malaria burden(4). 74\% of investments in 2016 were spent in the WHO African Region, followed by the WHO regions of South-East Asia (7\%), the Eastern Mediterranean and the Americas (each 6\%), and the Western Pacific (4\%)(8).

In Ethiopia, More than 75 percent of the total area of is malarious and over 63 million people live in these areas, making malaria the leading public health problem in the country. Benshangul Gumuz, Gambela, Tigray, and Amhara Regional States are among the regions with the highest proliferation of the disease(9). Ethiopia highlands are home to the majority of the country's population where the cooler climate serving as a natural buffer against malaria transmission. Now, new data show that increasing temperatures over the past 35 years are eroding this buffer, allowing conditions more favorable for malaria to begin climbing into highland areas $(10,11)$. There was a clear decrease in the malaria incidence rate and malaria death rate in Ethiopia from 2001 to 2016; however, malaria case number and incidence remained high, 19.8 cases per 1000 people reported in 2016 (12) which exceeds the WHO standard for pre-elimination(13).

Malaria remains to be the major public health challenge in the Amhara Region (14). Among ten zones and three town administrations in the Amhara region, West Gojjam Zone accounts for one-third of the malaria burden of the region. This zone constitutes 13 districts, among which half of the malaria burden exists just in three districts: Wonberma (24\%), Burie-Zuria (13\%), and Jabitehnan (12\%)(15).

Malaria incidence varies among countries as well as among the different regions of a given country. These differences can be attributed to climatic (meteorological variability), environmental (community level factor), host factors (individual level), socio-demographic, underutilization of interventions, weak monitoring and evaluation system, gaps in service delivery, lack of supply, and health information systems, and economic factors (16-18).

The understanding of the epidemiology and the disease pattern in time and space is needed to manage the disease. Thus, the identification of geographical areas using geographic information systems and spatiotemporal statistical analyses has become indispensable for proper allocation and mobilization of resources. However, there is scanty of information on district level spatial, temporal and spatiotemporal malaria variation of malaria incidence and risk factors in West Gojjam Zone. Therefore, the aim of this study was to assess spatial, temporal and spatiotemporal distribution patterns and risk factors associated to count of malaria incidence in West Gojjam Zone from 1 July 201330 June 2018.

\section{Methods}

\section{Study area}

The study was conducted in West Gojjam Zone of the Amhara Region. The study area, West Gojjam, is located in the Northwestern and north-central parts of Ethiopia. The town of this zone is Finote Selam which is 246 and 173 kilometers far from Addis Ababa, the capital of Ethiopia and the city of Amhara region, Bahir Dar respectively. West Gojjam is bordered on the south by the Abay River which separates it from the Oromia Region and BenishangulGumuz Region, on the west by Agew Awi, on the northwest by North Gondar, on the north by Lake Tana and the Abay River which separates it from the South Gondar, and on the east by East Gojjam. The digital elevation model indicates that the lowest and the highest peaks of the area are 684 and 3592 meters above sea level (Figure 1).

\section{Study design and period}


This time series cross sectional study design was conducted from 1 July 2013 to 30 June 2018 using a malaria report obtained from the Amhara Public Health Institute and climatic data obtained from West Amhara Meteorology Agency.

\section{Data}

Data on malaria were obtained from weekly reports from Amhara Public Health Institute. The data were reported from health facilities to the district health offices in weekly surveillance forms. District health offices report it to zonal health departments and then to Amhara Institute of Public Health. The malaria datasets were aggregated at a district levels and comprised information on malaria cases, type of parasites (P.falciparum, P.vivax), age category and time of illness (week and year). Climatic data obtained from West Amhara Meteorology Agency.

The spatial coordinates (the latitudes and longitudes) for each district were obtained from the Central Statistical Agency's polygon shape file. The spatial data were created in ArcGIS10.2.2 for each district. The population data were used to calculate annual malaria incidence and used as known underlying population at risk to fit Poisson model. Population data for each district for each year was obtained from Central Gondar Zone Health Department.

\section{Data management and analysis}

All 13 districts in West Gojjam zone were included and geo-coded for this study. A shape file with district boundaries and polygon shapes were obtained from the Amhara Region Central Statistical Agency (CSA) and each district was geo-referenced to its geographic centroid. The spatial data was created in ArcGIS10.2.2 version software for each district.

Weekly and annual cumulative malaria incidences of each district were calculated and plotted to check the annual fluctuations of malaria transmission from 1 July 2013 to 30 June 2018. The number of malaria cases to the population at risk was used to calculate the monthly and annual cumulative malaria incidences during the specified period.

The discrete Poisson model: the discrete poison model was used because the number of cases in each location was Poisson distributed and the nature of the data was count(19) and the population was the combined number of personyears lived used to fit the Poisson model.

Cluster analysis: ArcGIS10.2.2 was used to merge malaria data to the shape file and to test the hypothesis of the malaria distribution pattern using Global Moran’s I, and hot spot analysis using (Getis-Ord Gi* statistic) was conducted.

The scan statistics developed by Kulldorff using SaTScan ${ }^{\text {TM }} 9.6$ software was used to identify the presence of the purely spatial, temporal, and space-time malaria clusters. The scan statistics did scanning gradually across time and/or space to identify the number of observed and expected observations inside the window at each location. The scanning window was an interval (in time), a circle (in space) or a cylinder with a circular base (in space-time) to which window sizes were determined, and the window with the maximum likelihood was the most likely cluster, and a p-value was assigned to this cluster.

Purely spatial clusters: This spatial statistical analysis method employs the creation of a circular window that scans the entire study area. The radius of the circle varies continuously from zero to a specified maximum size. The maximum-size specified the percentage of population at risk within the scanning window. Since researchers 
recommend the maximum-size be no greater than $50 \%$, that is a reported cluster can contain at most $50 \%$ of the total population at risk(20), so maximum cluster size used was $50 \%$ of the population at risk. The null hypothesis is that disease risk is the same inside and outside the scanning window in space, while the alternative hypothesis is that the risk within the window is different from that outside the window. For this study, the spatial window of 1 and 40 kilometer were used. The circle with the maximum likelihood ratio and containing more cases than expected is identified as the most likely (primary) cluster that is least likely to have occurred by chance(19). The likelihood ratio for this window comprises the maximum likelihood ratio test statistic. The p-value was estimated using Monte Carlo simulations (999), by comparing the rank of the maximum likelihood from the real dataset with the maximum likelihoods from the random datasets(20). A significance level of alpha $<0.05$ was used to test whether the cluster was significant.

Spatiotemporal clusters: The space-time scan statistic was employed to detect clusters in both space and time. This helped us to detect clusters that were not detected by the purely spatial statistic. To detect spatiotemporal clusters a cylindrical window was used. The base of the cylinder represents space, as in the purely spatial scan statistic, while the height reflects the time. For this study, the time window was restricted to the annual and the spatial window to 1 kilometer. Districts with a significant number of cases within the corresponding time were identified using a p-value that was determined using Monte Carlo simulations. For purely spatial and space- time analyses, secondary clusters in addition to the most likely cluster (primary) were identified using an iterative manner as described in Kulldorff(21) for each purely spatial and space-time scan statistics. This procedure was then repeated until no clusters remained with $p$-values of less than 0.05 . The maximum cluster size was set to $50 \%$ of the population at risk. Purely temporal scan statistics used a window that moved in one dimension only using the height of the cylindrical window as the time dimension. A p-value was generated using Monte Carlo simulations in a manner similar to the spatiotemporal clusters. A significance level of alpha $<0.05$ was used to identify a significant cluster.

Purely temporal clusters: temporal scan statistics used a window that moved in one dimension only using the height of the cylindrical window as the time dimension. A p-value was generated using Monte Carlo simulations like spatiotemporal clusters. Like spatial and spatiotemporal clusters, a significance level of alpha $<0.05$ were used to identify a significant risk period. For purely temporal analyses, only the most likely cluster was reported. The scan was used to scan for areas/districts and times/periods with high rate malaria clusters.

Statistical analyses were performed and reported using Excel, SaTScan ${ }^{\mathrm{TM}} 9.6$ and ArcGIS10.2.2 software. Excel spreadsheet was used to describe data, draw line graphs and pie charts. Spatial and spatiotemporal clusters were analyzed using SaTScan ${ }^{\mathrm{TM}} 9.6$ programs. The map of significant clusters was plotted using ArcGIS 10.2.2 software.

\section{Correlation and regression analysis}

Spearman's correlation, bivariate, and multivariable negative binomial regressions were performed to test the relationship between climatic factors and count of malaria incidence. Correlation analysis was done considering 0,1 , and 2 lag months of the climatic variables and count of malaria incidence. The multicollinearity between climate variables using the Variance Inflation Factor (VIF) was checked before the multivariable regression analysis and was found to be $₫ 5$.

A bivariate negative binomial regression was performed to examine the crude associations between the climatic factors and the outcome variable and variables with $p$-value $\leq 0.2$ were entered into the final model. In the multivariable negative binomial regression model, adjusted Incidence Rate Ratio (IRR) with 95\% confidence interval was calculated to identify the independent effect of each explanatory variable with the outcome variable. A 
significance level of 0.05 was considered for all statistical tests. Adjusted IRR with $90 \% \mathrm{Cl}$ was used to declare statistical significance. Statistical analyses were conducted using SPSS 20.

\section{Ethical Clearance}

Ethical clearance was obtained from the Research and Ethical Review Board of Institute of Public Health, College of Medicine and Health Sciences, University of Gondar. Support letters were obtained from Amhara Public Health Institute, West Amhara Meteorology Agency, and Central Gondar Zone Health Department for retrieving retrospective data from records. All the information was kept confidential and no individual identifiers were collected.

\section{Results}

\section{Distribution of malaria infections}

From 13 districts of West Gojjam, a total of 342,947 malaria cases were reported from 1 July, 2013 to 30 June 2018. Plasmodium falciparum 58.76 \%(201,510) was the dominant species followed by Plasmodium vivax $41.24 \%($ $141,437)$. The highest proportion, $69.33 \%(237,753)$ of the malaria cases was accounted by $\geq 15$ years of age, followed by $18.38 \%(63,049)$ which was accounted by $5-14$ years of age. The $<5$ accounted $12.29 \%(42,145)$ of malaria cases.

The average cumulative annual malaria incidence was 29.13 per 1000 population at risk. The highest cumulative annual malaria incidence 144.4 occurred in Jabitenan district in 2015/16. Whereas, the lowest incidence 3 per 1000 population at risk occurred in Bahir Dar Zuriya and Mecha districts in 2017/18 and 2016/17 respectively (Figure 2).

\section{Trends of malaria infections}

There was a decreasing trend of malaria incidence and shows weakly and seasonal variability. The period from 1 July 2013 to 30 June 2014 had shown multiple weekly peaks of malaria case incidence.

There were two peak malaria case incidence seasons for each year. The first peak was from week 16 through week 28 and the second peak was from about week 37 to week 47 . Fluctuating temporal trends of annual malaria incidence were observed (Figure 3).

\section{The spatial pattern of malaria incidence using ArcGIS10.2.2}

\subsubsection{Global spatial autocorrelation}

The global autocorrelation results indicated that the malaria incidence was clustered (Global Moran's I=0.303613, P value $=0.025485$ ) (Figure 4).

\section{Spatial, temporal and spatiotemporal SaTScan clusters}

\section{High rate spatial clusters}


Malaria distribution was found to be clustered. Five high rate spatial clusters were detected throughout the study period. Jabitenan (LLR=59178.27, $p<0.001$ ), Quarit (LLR=7821.767, $p<0.001)$, Sekela ( $L L R=843.9762, p<0.001)$, Bure $(L L R=81.49807, p<0.001)$, and Wonberma (LLR=77.73337, $p<0.001)$ districts were high rate malaria clusters identified hierarchically. (Table 1, Figure 5)

Table 1: Significant high rate spatial clusters of malaria in West Gojjam, 1 July 2013-30 June 2018

\begin{tabular}{|c|c|c|c|c|c|c|c|}
\hline Cluster & District & POP. & Coordinates/ radius & Obs.* & Exp.* & RR & LLR \\
\hline 1 & Jabitenan & 255261 & $\begin{array}{l}10.700600 \mathrm{~N}, 37.276100 \mathrm{E} / 0 \\
\mathrm{~km}\end{array}$ & 107306 & 34136.29 & 4.12 & 59178.27 \\
\hline 2 & Quarit & 132459 & $\begin{array}{l}10.974000 \mathrm{~N}, 37.441100 \mathrm{E} / 0 \\
\mathrm{~km}\end{array}$ & \begin{tabular}{|l|}
36070 \\
\end{tabular} & 17713.88 & 2.16 & 7821.767 \\
\hline 3 & Sekela & 160710 & $\begin{array}{l}10.998700 \mathrm{~N}, 37.207900 \mathrm{E} / \\
0 \mathrm{~km}\end{array}$ & \begin{tabular}{|l|}
27562 \\
\end{tabular} & 21491.82 & 1.31 & 843.9762 \\
\hline 4 & Bure & 179122 & $\begin{array}{l}10.541900 \mathrm{~N}, 37.048900 \mathrm{E} / \\
0 \mathrm{~km}\end{array}$ & 25883 & 23954.14 & 1.09 & 81.49807 \\
\hline 5 & Wonberma & 124328 & $\begin{array}{l}10.489000 \mathrm{~N}, 36.79970 \mathrm{E} / 0 \\
\mathrm{~km}\end{array}$ & 18218 & 16626.53 & 1.1 & 77.73337 \\
\hline
\end{tabular}

P-value $<0.001$ for all clusters, POP. = Population, RR, =Relative Risk, LLR= Log Likelihood Ratio, Obs.*= Number of observed cases in a cluster, Exp. ${ }^{*}=$ Number of expected cases in a cluster.

\section{High rate temporal clusters}

Significantly a high rate of purely temporal malaria cluster was observed in this study from 1 July 2013-30 June 2015 $(\mathrm{LLR}=22448.54, \mathrm{p}<0.001)$ (Table 2).

Table 2: Significant high rate temporal cluster of malaria in West Gojjam, 1 July 2013-30 June 2018

\begin{tabular}{|l|l|l|l|l|l|l|l|}
\hline Cluster & District & Time frame & Obs.* & Exp. $*$ & RR & LLR & p-value \\
\hline 1 & All & $2013 / 7 / 1$ to $2015 / 6 / 30$ & 195144 & 133649 & 2.07 & 22448.54 & 0.001 \\
\hline \\
RR=Relative Risk, LLR=Log Likelihood Ratio, Obs.*=Number of observed cases in a cluster, Exp.*=Number of expected cases in \\
a cluster.
\end{tabular}

\section{High rate spatiotemporal clusters}

The spatiotemporal analysis provided further evidence for a higher than expected number of malaria cases arising within a defined place and time. Significant spatiotemporal malaria clusters were detected at Jabitenan between 1 July 2014 to 30 June 2016, Quarit and Sekela between 1 July 2013 to 30 June 2015, Wonberma, Bure, Bahir Dar Zuriya, GonjiKolela, South Achefer, Yilmana Densa, and North Achefer between 1 July 2013 to 30 June 2014 and Dembecha between 1 July 2015 to 30 June 2016(Table 3).

Table 3: Significant high rate spatiotemporal clusters of malaria in West Gojjam, 1 July $2013-30$ June 2018 


\begin{tabular}{|l|l|l|l|l|l|l|l|}
\hline Cluster & District & Time frame & Obs.* & Exp.* & RR & LLR & p-value \\
\hline 1 & Jabitenan & $2014 / 7 / 1$ to $2016 / 6 / 30$ & 62712 & 13550.07 & 5.44 & 50789.1 & $<0.001$ \\
\hline 2 & Quarit & $2013 / 7 / 1$ to $2015 / 6 / 30$ & 19476 & 6907.49 & 2.93 & 7857.882 & $<0.001$ \\
\hline 3 & Wonberma & $2013 / 7 / 1$ to $2014 / 6 / 30$ & 9604 & 3214.69 & 3.04 & 4182.239 & $<0.001$ \\
\hline 4 & Bure & $2013 / 7 / 1$ to $2014 / 6 / 30$ & 11284 & 4612.85 & 2.5 & 3489.048 & $<0.001$ \\
\hline 5 & Sekela & $2013 / 7 / 1$ to $2015 / 6 / 30$ & 16051 & 8384.58 & 1.96 & 2845.244 & $<0.001$ \\
\hline 6 & Bahir Dar Zuriya & $2013 / 7 / 1$ to $2014 / 6 / 30$ & 10981 & 5715.51 & 1.95 & 1946.236 & $<0.001$ \\
\hline 7 & Gonji Kolela & $2013 / 7 / 1$ to $2014 / 6 / 30$ & 6627 & 3164.35 & 2.12 & 1453.762 & $<0.001$ \\
\hline 8 & South Achefer & $2013 / 7 / 1$ to $2014 / 6 / 30$ & 6033 & 4032.82 & 1.5 & 435.6952 & $<0.001$ \\
\hline 9 & Yilmana Densa & $2013 / 7 / 1$ to $2014 / 6 / 30$ & 7978 & 6868.12 & 1.17 & 87.03831 & $<0.001$ \\
\hline 10 & Dembecha & $2015 / 7 / 1-2016 / 6 / 30$ & 4529 & 4111.62 & 1.1 & 20.75957 & $<0.001$ \\
\hline 11 & North Achefer & $2013 / 7 / 1-2014 / 6 / 30$ & 6705 & 6297.32 & 1.07 & 13.16705 & $<0.001$ \\
\hline $\begin{array}{l}\text { RR=Relative risk, LLR=Log likelihood ratio, Obs. }{ }^{*}=\text { Number of observed cases in a cluster, Exp.*=Number of expected cases in a } \\
\text { cluster. }\end{array}$ & & & & & \\
\hline
\end{tabular}

\section{Spearman's correlation analysis}

Correlation analysis was conducted to quantify the relationship between monthly malaria incidence and climatic variables during the study period, with 0,1 and 2 months lag. A significant positive correlation was found between monthly average temperature and the monthly count of malaria incidence at 0,1 , and 2 months lag. The correlation coefficient decreased as the number of lags increased from 0 to 2 months. Monthly mean rainfall and number of monthly malaria cases showed a negative correlation at all lags $(0,1$, and 2 months). All lag-months were significant and the correlation increased as the number of lags increased. Monthly mean relative humidity was inversely correlated with the number of malaria cases (ranged from -0.334 to -0.347) (Table 4).

Table 4: Correlation between the monthly counts of malaria incidence and mean monthly climatic variables in West Gojjam, 1 July 2013-30 June 2018 


\begin{tabular}{|l|l|l|l|}
\hline Monthly mean climatic variables & Lag-months & Spearman's r & p-value \\
\hline Temperature $\left({ }^{\mathrm{O}} \mathrm{C}\right)$ & 0 months & 0.318 & 0.01 \\
\cline { 2 - 4 } & 1 month & 0.284 & 0.01 \\
\cline { 2 - 4 } & 2 months & 0.209 & 0.01 \\
\hline Rainfall (mm) & 0 months & -0.195 & 0.01 \\
\cline { 2 - 4 } & 1 month & -0.311 & 0.01 \\
\cline { 2 - 4 } & 2 months & -0.332 & 0.01 \\
\hline Relative humidity (\%) & 0 months & -0.334 & 0.01 \\
\cline { 2 - 4 } & 1 month & -0.391 & 0.01 \\
\cline { 2 - 4 } & 2 months & -0.347 & 0.01 \\
\hline
\end{tabular}

\section{Regression analysis}

Multivariable regression results show that monthly average temperature, monthly average rainfall, and monthly average relative humidity were statistically associated with the rate of malaria incidence. There was a significant positive association between monthly average temperature and malaria incidence for all lag months. Monthly average rainfall and malaria incidence were negatively associated with all lag months. There was a significant inverse relationship between relative humidity and malaria at 2 months lag.

There was a significant positive association between monthly average temperature (Adjusted IRR $=1.301 ; 95 \% \mathrm{Cl}$ $1.212,1.396)$ and the count of malaria incidence at 0 lag months. This means that $1{ }^{0} \mathrm{C}$ rise in monthly average temperature increases count of malaria incidence by $30.1 \%$ (95\% $\mathrm{Cl} 21.2 \%, 39.6 \%)$. Monthly average rainfall (Adjusted IRR $=0.998 ; 95 \% \mathrm{Cl} 0.996,0.999)$ was inversely associated with a count of malaria incidence at 0 lag months. There was a significant positive association between monthly average relative humidity and count of malaria incidence (Adjusted IRR=1.021; 95\%Cl 1.009, 1.033) at 2 lag months (Table 5).

Table 5: Negative binomial regression analysis of the effect of climate variability on malaria in West Gojjam, 1 July 2013-30 June 2018 


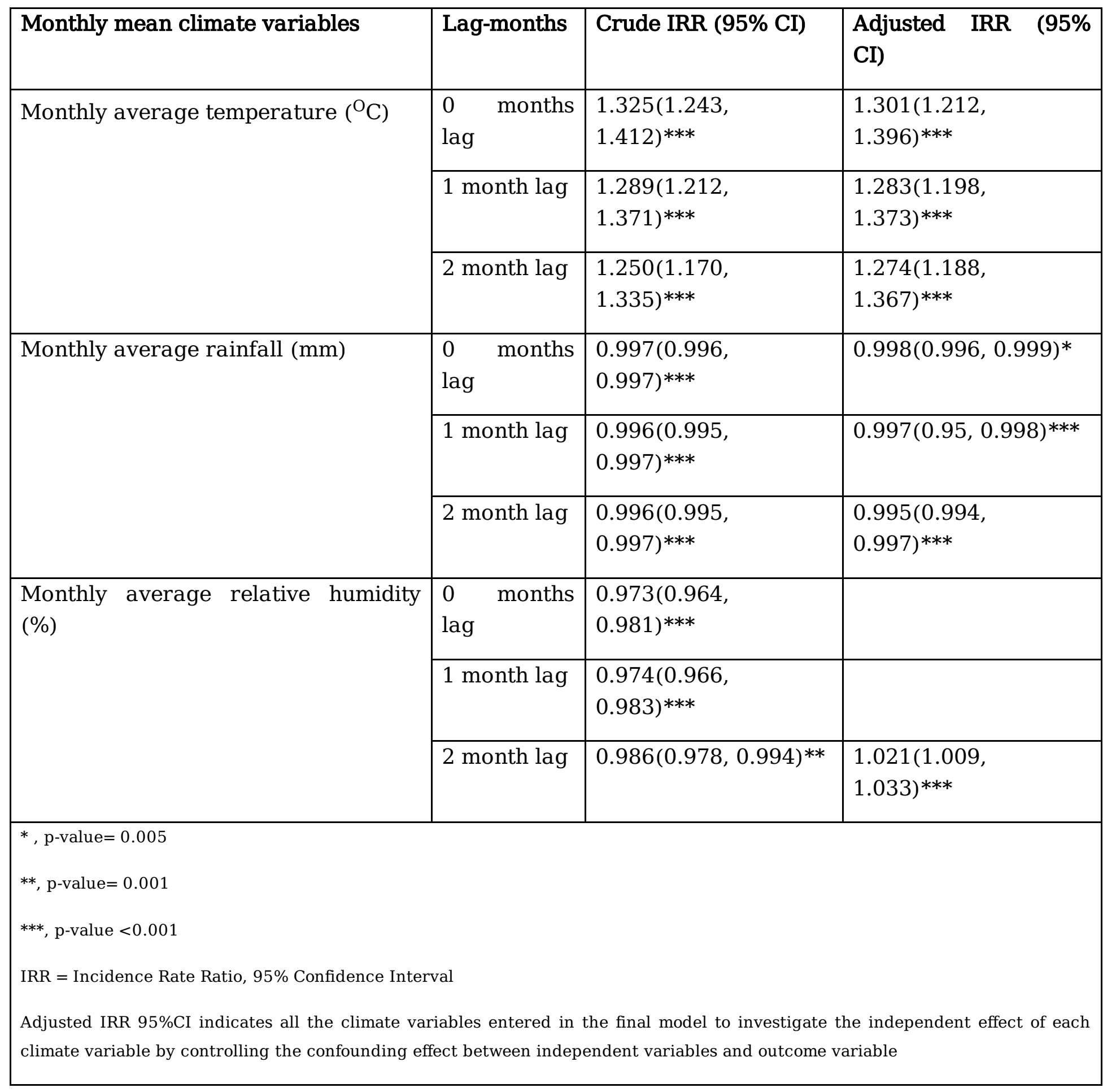

\section{Discussion}

Malaria incidence had shown interannual variability across districts and survey years in West Gojjam zone from 1 July 2013-30 June 2018. Fluctuating seasonal temporal trends of malaria incidence were observed. All districts reported malaria cases during the study period.

The average annual cumulative malaria incidence proportion was $2.91 \%$. Thus, the result was lower than studies conducted in other parts of northwest Ethiopia 30\%(22), village-level study conducted in Dabat district(9.7\%) (23), in rural SNNP region (6.8\%)(24), Ethiopia's 2017 national malaria report (4.5\%)(25), 2011 (43\%) and in $2014(33 \%)$ in Tigray Regional State(26). Also, it has shown a decreasing trend year after year during the study period from 48.13 in 
1 July 2013-30 June 2014, to 14.15 in 1 July 2017-30 June 2018 per 1,000 populations at risk. This variation might be due to the time period of this study, which is after the first National Strategic Plans (NSP 2006-2010)and during the second five-year National Strategic Plans for Malaria Prevention, Control, and Elimination (NSP 2011-2015)(27), and endorsement of malaria elimination in 2016 using different surveillance mechanisms, and mobilizing communities (28). As well, malaria control programs that the country has been carried out previously in collaboration with partners brought a good lesson in training human power, provision of services, indoor residual spraying, improve surveillance activities and laid the foundation towards reducing malaria. The treatment, the controlling method, and the people awareness is improved now compared to the past(9).

The average annual cumulative malaria incidence(2.91\%) was similar to the study conducted in Ethiopia from 2001 to $2016(2.9 \%)(12)$ but it is still higher than the WHO pre-elimination standard ( $\mathbb{1} 1$ case/1,000 population at risk/year) (13). This might be due to the knowledge, attitude and practice of the community towards malaria prevention and control options are still at a low level(29), multiple mosquito breeding sites would be difficult for environmental management, malaria control methods like indoor residual spraying of houses may not be performed timely, and usually sprayed after the outbreak begun, LLINs may not be replaced timely and not replaced at all, and the low practice of environmental management by the community and health extension workers might be the possible explanations for the observed level of average cumulative annual malaria incidence(15).

Purely spatial high rate malaria clusters were observed in Jabitenan, Quarit, Sekela, Bure, and Wonberma districts hierarchically. These clusters occurred in a specific geographical area. This might be because they share similar geographical parameters, such as altitude and weather conditions, and economic characteristics(30). Since the importance of cluster analysis is to detect the aggregation of disease cases and to find evidence of risk factors on which prevention and control activities can be focused(31), it would be good to give priority intervention to clusters identified in this study

Significantly a high rate of purely temporal malaria cluster was observed from 1 July 2013 to 30 June 2015 $(L L R=22448.54, p<0.001)$. There was a significant decrease in malaria incidence after this two year risk period. This might be due to the country is moving forward to eliminate malaria in the country by 2030 from 2016 onwards by training human power, provision of services, indoor residual spraying, improved surveillance activities compared to the time before 2016(28).

The spatiotemporal analysis provided further evidence for a higher than expected number of malaria cases arising within a defined place and time. Significant spatiotemporal malaria clusters were detected at Jabitenan from 1 July 2014 to 30 June 2016, Quarit and Sekela from 1 July 2013 to 30 June 2015, Wonberma, Bure, Bahir Dar Zuriya, GonjiKolela, South Achefer, Yilmana Densa, and North Achefer from 1 July 2013 to 30 June 2014, and Dembecha from 1 July 2015 to 30 June 2016. Space-time clusters from 1 July 2013 to 30 June 2015 might be explained by their occurrence preceding the endorsement of national malaria elimination road map(28). Those space-time clusters which occurred after 30 June 2015 may be due malaria intervention measures might have not been taken appropriately, or the interventions might have not been utilized correctly $(29,32)$ and risk from hunger, disease and lack of water exacerbated by El Niño(33).

This implies that a seasonal and unstable malaria transmission is the characteristics of the districts. Thus, decisionmakers and health managers need to maintain the quality interventions consistently to prevent the cyclic resurgence of epidemics.

A trend of seasonality in peak malaria cases, which occurred more frequently from September to December and March to June, was observed. This finding is consistent with previous studies in the northwest part of Ethiopia $(22,32,34)$

Page $11 / 18$ 
that showed climate factors significantly affected seasonal malaria incidence. Peak malaria transmission occurs between September and December in most parts of Ethiopia, after the main rainy season from June to July. Besides, some areas experience a second minor malaria transmission period from April to June, following a short rainy season from March to May with high maximum temperature $(35,36)$. The rainy season ends leaving stagnant water collections and during dry season rivers start pooling making the environment favorable for malaria vector breeding(37).

Temperature was statistically significant and positively associated to count of malaria incidence in this study. The finding of this study is in line with previous studies of Jimma town(3), and Amhara region(38). Again it is supported by a study done in Yunnan Province, China which showed that annual average temperature was positively associated with the malaria incidence rate, according to the GWR model(39). Temporal correlation analysis between malaria and meteorological factors in Motuo County, China revealed that temperature was an important factor in the transmission of malaria(40). Spatiotemporal data at a regional scale in highlands of Colombia and Ethiopia showed spatial distribution of the disease changes with the interannual variability of temperature which provided evidence for an increase in the altitude of malaria distribution in warmer years, which implies that climate change, without mitigation, will result in an increase of the malaria burden. Temperature is known to influence transmission intensity through its effects growth of the mosquito vector and pathogen development within the vector(41).

The effect of rainfall on malaria incidence was found to be inversely correlated. This finding is in argument to previous studies of Chennai, India(42), Yunnan Province, China(39), Motuo County, China(40), and Jimma town(3). This might be due to the strength of rainfall vary in different settings to be able to erode and outflow mosquito breeding sites. Water collections that support vector breeding appear mainly after the rains, and therefore malaria transmission is highest following the rainy season in Ethiopia(43). The presence of high rainfall may not contribute for the rise of malaria incidence as it causes overflow and erodes water collections. It might drain pooling rivers during dry seasons as well. Malaria starts getting higher immediately after the rainy season ends. Therefore, this implies rainfall is a good indicator for malaria prevention and control activities.

\section{Conclusion}

This study identified spatial, temporal, spatiotemporal clustering and seasonal pattern of malaria incidence. There was also a decreasing temporal trend of malaria incidence. Mean monthly temperature and rainfall were directly and inversely associated to malaria incidence respectively at all lag moths. Relative humidity was positively associated at 2 lag months. Considering these space-time variations and factors would be useful for the prevention and control and ultimately achieve elimination targets.

\section{Declarations}

\section{Ethics approval and consent to participate}

Ethical clearance was obtained from Research and Ethical Review Committee of Institute of Public Health, College of Medicine and Health Sciences, University of Gondar. There were no individual participants in the study because it was secondary data/count of malaria which has no personal identifiers.

\section{Consent for publication}

Not applicable. 


\section{Availability of data and material}

Weekly malaria data was taken from Amhara Public Health Institute, climate data from West Amhara Meteorological Agency, projected population from Central Gondar Zone Health Department, and Amhara Region Central Statistical Agency.

\section{Competing interests}

No competing interest

\section{Funding}

Fund was obtained from Debre Markos University.

\section{Authors' contributions}

Eniyew Tegegne conceived and initiated the study and made data available. All authors made major contributions to the study design, statistical analysis and writing of the manuscript and approved the submitted version of the manuscript.

\section{Acknowledgments}

We are thankful to Amhara of Public Health Institute, West Amhara Meteorological Agency, Amhara Region Central Statistical Agency and Central Gondar Zone Health for letting us the required data for the study.

\section{References}

1. World Malaria report 2014: Summary 2015 [01/02/2019]. Available from: https://www.who.int/malaria/publications/world_malaria_report_2014/en/.

2. Feng J, Liu J, Feng X, Zhang L, Xiao H, Xia ZJTAjotm, et al. Towards malaria elimination: monitoring and evaluation of the "1-3-7" approach at the China-Myanmar border. 2016;95(4):806-10.

3. Alemu A, Abebe G, Tsegaye W, Golassa LJP, vectors. Climatic variables and malaria transmission dynamics in Jimma town, South West Ethiopia. 2011;4(1):30.

4. World malaria report 2018. 2018 [01/02/2019]. Available from: https://www.who.int/malaria/publications/worldmalaria-report-2018/en/.

5. Pavli A, Maltezou, Helena.Travel medicine infectious disease. Malaria and travellers visiting friends and relatives. 2010;8(3):161-8.

6. Schumacher R-F, Spinelli. Malaria in children. Mediterranean journal of hematology. 2012;4(1).

7. Global technical strategy for malaria 2016-2030 2015 [02/02/2019]. Available from: https://apps.who.int/iris/bitstream/handle/10665/176712/9789241564991_eng.pdf?sequence=1.

8. Key points: World malaria report 2017. 2017 [23/05/2019]. Available from:

https://www.who.int/malaria/media/world-malaria-report-2017/en/.

9. Improved Controlling Methods Help to Reduce Malaria: Report from Government of Ethiopia. 2018 [23/05/2019]. Available from: https://reliefweb.int/report/ethiopia/improved-controlling-methods-help-reduce-malaria.

10. F. Malaria risk increases in ethiopian highlands as temperatures climb. 2017 [01/01/2019]. Available from: https://www.google.com/search?client=firefox-bab\&q=Fiondella+F.+Malaria+Risk+Increases+in+Ethiopian+Highlands+as+Temperatures+Climb.+2017. 
11. Scott M. Malaria risk zones expand to higher elevations in Ethiopian Highlands. 2017 [01/02/2019]. Available from: https://climate.gov/news-features/featured-images/malaria-risk-zones-expand-higher-elevations-ethiopianhighlands.

12. Taffese HS, Hemming-Schroeder E, Koepfli C, Tesfaye G, Lee M-c, Kazura J, et al. Malaria epidemiology and interventions in Ethiopia from 2001 to 2016. 2018;7(1):103.

13. Country Classification by Malaria Elimination Phase. 2012. [03/06/2019.]. Available from: https://www.who.int/malaria/mpac/feb2012/elimination_classification.pdf.

14. Emerson PM, Ngondi J, Biru E, Graves PM, Ejigsemahu Y, Gebre T, et al. Integrating an NTD with one of "the big three": combined malaria and trachoma survey in Amhara Region of Ethiopia. 2008;2(3):e197.

15. Addisu Workineh Kassa, Mulugojjam Andualem Tamiru, Yeshanew. aAG. Assessment of Control Measures and Trends of Malaria in Burie-Zuria District,West Gojjam Zone, Amhara Region, NorthWest Ethiopia. 2015.

16. Van Lieshout M, Kovats R, Livermore M, Martens PJGEC. Climate change and malaria: analysis of the SRES climate and socio-economic scenarios. 2004;14(1):87-99.

17. Mogeni P, Williams TN, Fegan G, Nyundo C, Bauni E, Mwai K, et al. Age, spatial, and temporal variations in hospital admissions with malaria in Kilifi County, Kenya: a 25-year longitudinal observational study. 2016;13(6):e1002047.

18. Clements AC, Barnett AG, Cheng ZW, Snow RW, Zhou HNJMj. Space-time variation of malaria incidence in Yunnan province, China. 2009;8(1):180.

19. Kulldorff M. A spatial scan statistic. Communications in Statistics-Theory and methods. 1997;26(6):1481-96.

20. Kulldorff M, Nagarwalla N. Spatial disease clusters: detection and inference. Statistics in medicine. 1995;14(8):799-810.

21. Kulldorff M. An isotonic spatial scan statistic for geographical disease surveillance. Journal of the National Institute of Public Health. 1999;48(2):94-101.

22. Taddese AA, Baraki AG, Gelaye KA. Spatial modeling, prediction and seasonal variation of malaria in northwest Ethiopia. BMC Research Notes. 2019;12(1):273.

23. Alemu K, Worku A, Berhane Y, Kumie AJMj. Spatiotemporal clusters of malaria cases at village level, northwest Ethiopia. 2014;13(1):223.

24. Dessie DB. Spatial Modelling of Malaria Prevalence and Its Risk Factors in Rural SNNPR, Ethiopia: Classical and Bayesian Approaches. American Journal of Theoretical and Applied Statistics. 2017;6(6):254.

25. Ethiopia malaria operational plan. 2017. [3/6/2019]. Available from: http://www. pmigo v/docs/defau It-sourc e/defau It-docum ent-libra ry/malar ia-operationa I-plans /fy14/ethio pia_mop_fy14p df?sfvrs n=14Acc essed .

26. Gerensea H, Teklay H. Pattern and Trend of Malaria Morbidity and Mortality in Tigray Region, Ethiopia from 2011. 2017.

27. Ethiopian Public Health Institute. Ethiopia National Malaria Indicator Survey 2015. 2016 [03/06/2019].Availablefrom:https://ephi.gov.et/images/pictures/download2009/MIS-2015-Final-ReportDecember-_2016.pdf.

28. H. Ethiopia: AU Endorses Roadmap to Eliminate Malaria By 2030. 2017. [cited 03/06/2019]. Available from: https://allafrica.com/stories/201607200560.html.

29. Aderaw Z, Gedefaw M. Knowledge, attitude, and practice of the community towards malaria prevention and control options in anti-malaria association intervention zones of Amahara national regional state, Ethiopia. Global Journal of Medical Research. 2013.

30. Azage M KA, Worku A, Bagtzoglou AC (2015) Childhood Diarrhea Exhibits Spatiotemporal Variation in Northwest Ethiopia: A SaTScan Spatial Statistical Analysis. PLoS ONE 10 (12): e0144690. 
doi:10.1371/journal.pone.0144690.

31. Mboera LE, Senkoro KP, Mayala BK, Rumisha SF, Rwegoshora RT, Mlozi MR, et al. Spatio-temporal variation in malaria transmission intensity in five agro-ecosystems in Mvomero district, Tanzania. 2010.

32. Alemu K, Worku A, Berhane YJPo. Malaria infection has spatial, temporal, and spatiotemporal heterogeneity in unstable malaria transmission areas in northwest Ethiopia. 2013;8(11):e79966.

33. New York Times. Ethiopia El Niño Emergency - Fast Facts. 2016 [03/06/2019]. Available from: https://reliefweb.int/report/ethiopia/ethiopia-el-ni-o-emergency-fast-facts-june-2016.

34. Yeshiwondim AK, Gopal S, Hailemariam AT, Dengela DO, Patel HP. Spatial analysis of malaria incidence at the village level in areas with unstable transmission in Ethiopia. International Journal of Health Geographics. 2009;8.

35. Ethiopian malaria operational plan 2018. [03/06/2019]. Available from: https://www.pmi.gov/docs/defaultsource/default-document-library/malaria-operational-plans/fy-2018/fy-2018-ethiopia-malaria-operationalplan.pdf?sfvrsn=5.

36. Guide to the use of weather and climate information (WCI) for agricultural practices over selected district of Tigray, Ethiopia. National Meteorological Agency (NMA) of Ethiopia. 2017. [01/06/2019]. Available from: http://www.wmo.int/pages/prog/dra/raf/documents/Climate\%20Guide\%20for\%20Tigray\%20and\%20SNNPR.pdf.

37. Factors that Affect Malaria Transmission. [02/06/2019]. Available from: https://www.open.edu/openlearncreate/mod/oucontent/view.php?id=89\&printable=1.

38. Midekisa A, Beyene B, Mihretie A, Bayabil E, Wimberly MCJP, vectors. Seasonal associations of climatic drivers and malaria in the highlands of Ethiopia. 2015;8(1):339.

39. Yang D, Xu C, Wang J, Zhao YJBph. Spatiotemporal epidemic characteristics and risk factor analysis of malaria in Yunnan Province, China. 2017;17(1):66.

40. Huang F, Zhou S, Zhang S, Wang H, Tang L. Temporal correlation analysis between malaria and meteorological factors in Motuo County, Tibet. Malaria Journal. 2011;10(1):54.

41. Siraj AS-V, Mauricio, Bouma, MJ, Yadeta, D, Carrascal, D Ruiz, Pascual, M. Altitudinal changes in malaria incidence in highlands of Ethiopia and Colombia. 2014;343(6175):1154-8.

42. Kumar DS, Andimuthu R, Rajan R, Venkatesan MS. Spatial trend, environmental and socioeconomic factors associated with malaria prevalence in Chennai. Malaria journal. 2014;13(1):14.

43. Woyessa A, Deressa W, Ali A, Lindtjørn B. Malaria risk factors in Butajira area, south-central Ethiopia: a multilevel analysis. Malaria journal. 2013;12(1):273.

\section{Figures}




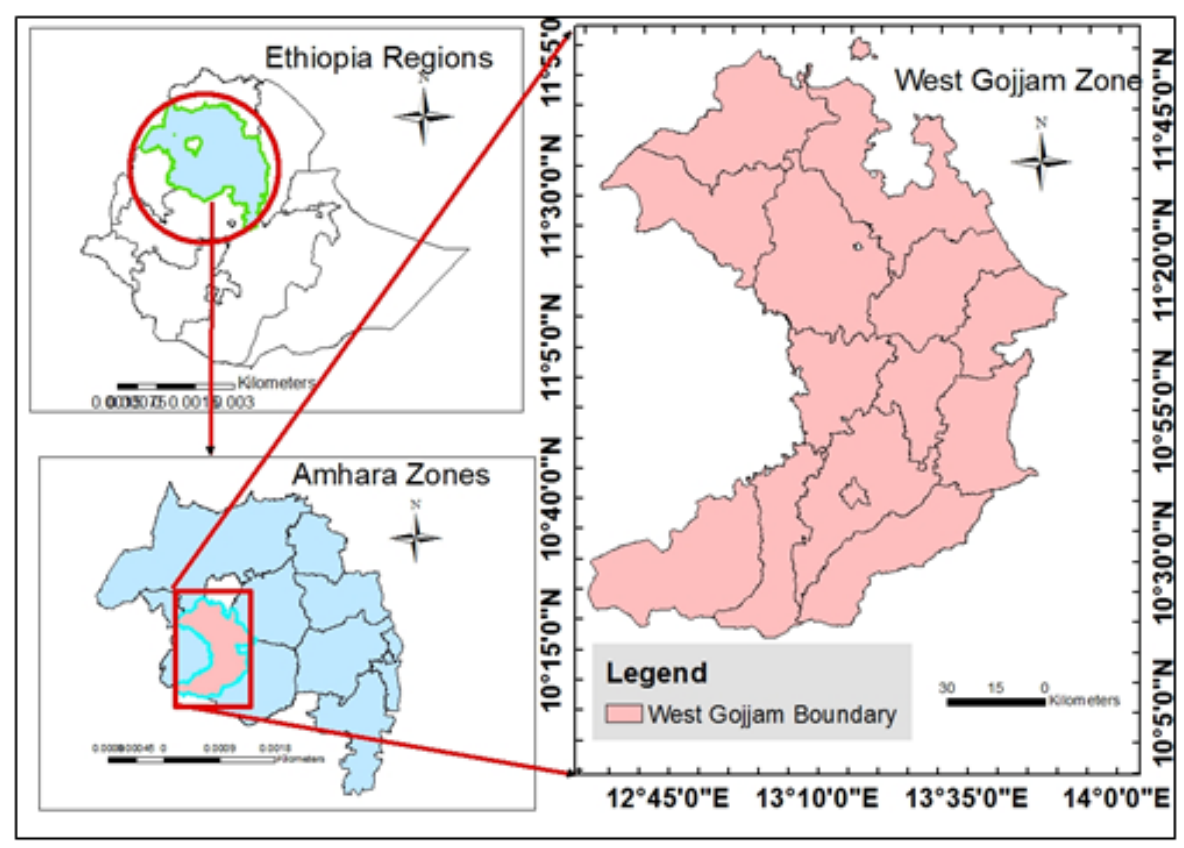

Figure 1

Location of West Gojjam/where the study was done.

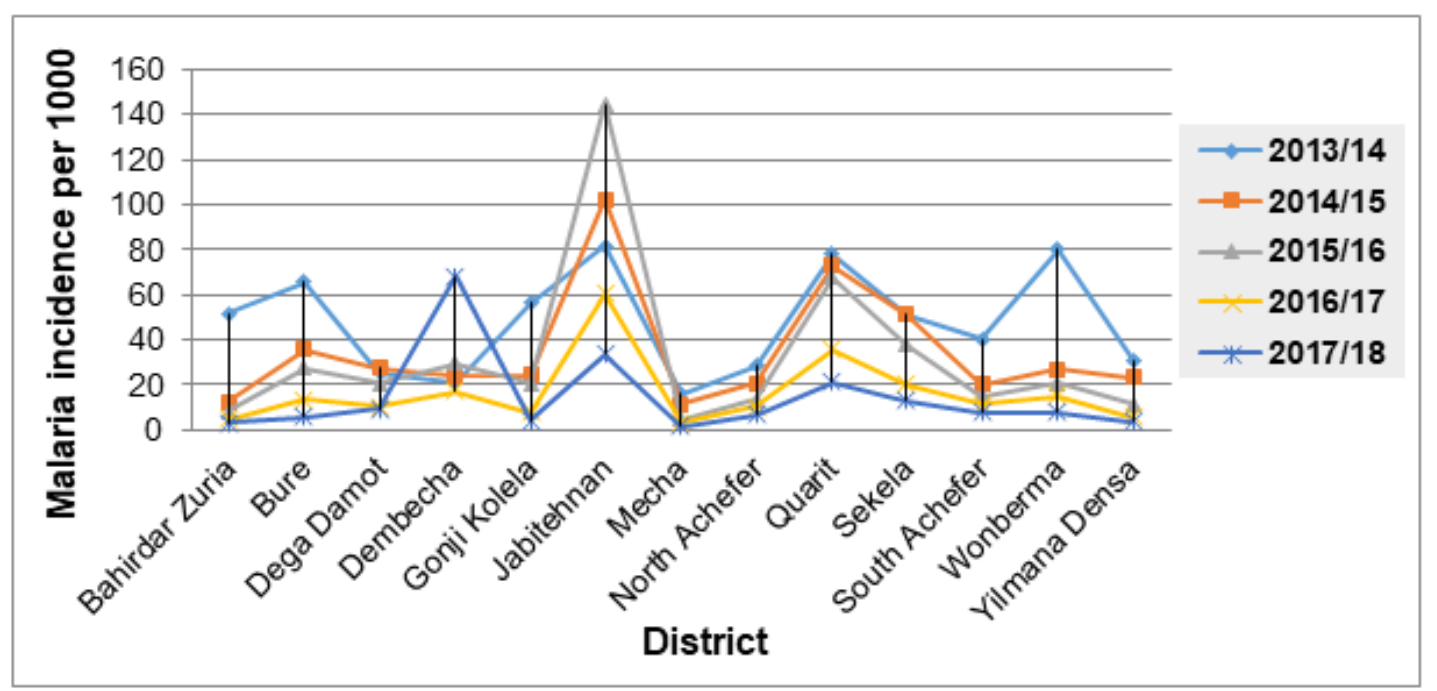

Figure 2

District level annual cumulative malaria incidence in West Gojjam, 1 July 2013-30 June 2018. 


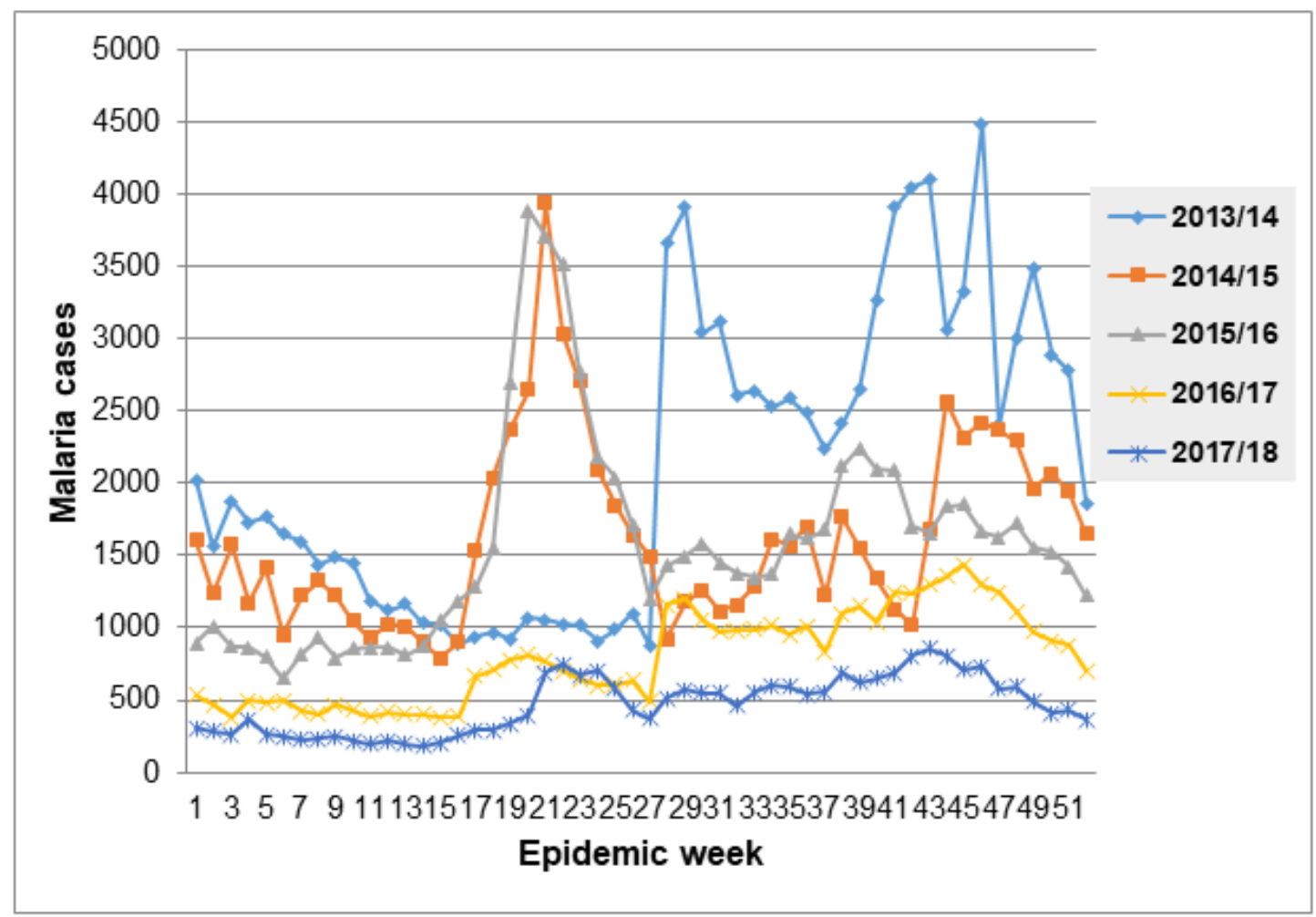

Figure 3

Temporal variation of malaria cases in West Gojjam, 1 July 2013-30 June 2018.

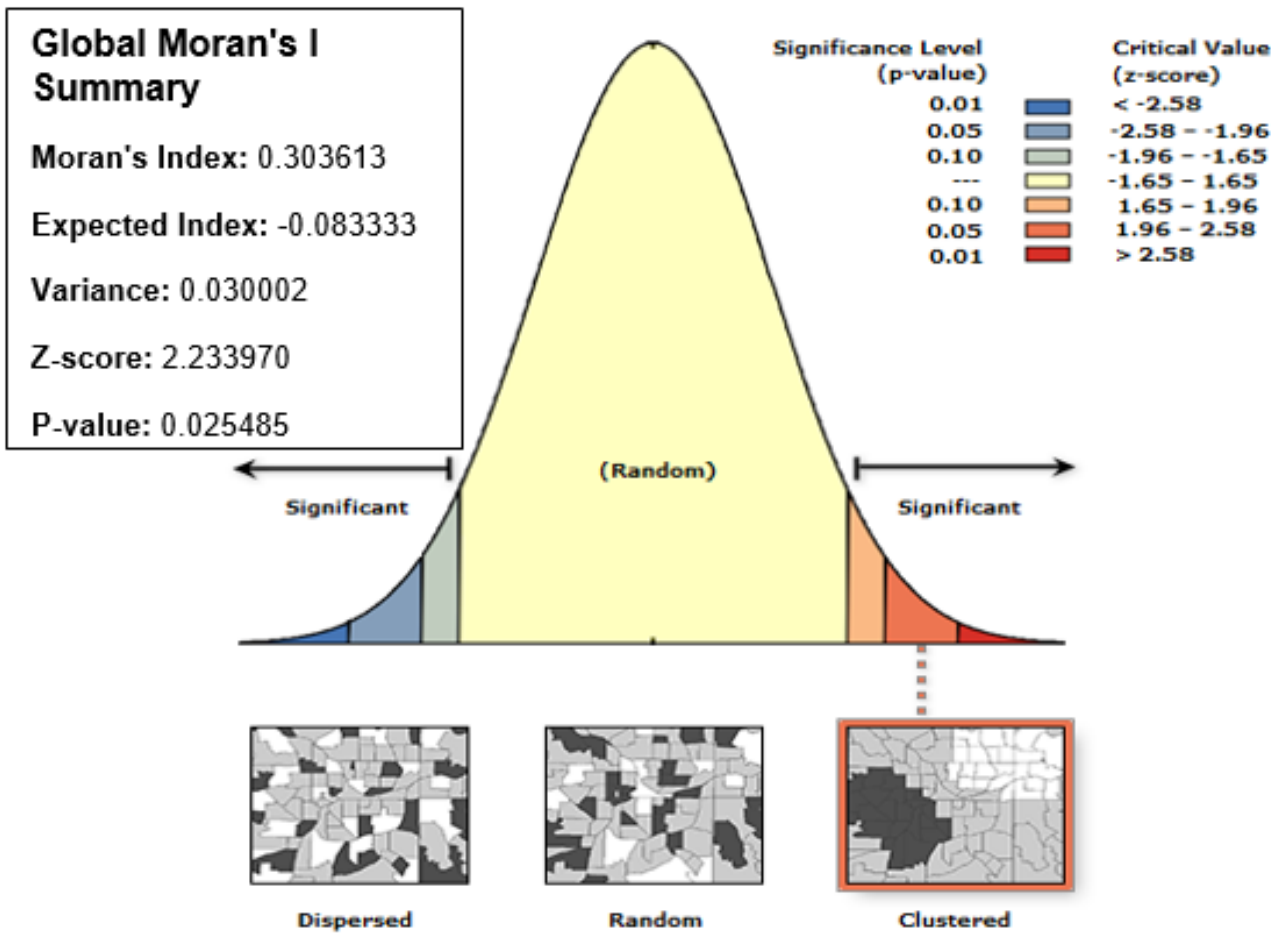

Figure 4

Spatial autocorrelation based on feature locations and attribute values (average cumulative annual malaria incidence) using the Global Moran's I statistic in West Gojjam, 1 July 2013-30 June 2018. 


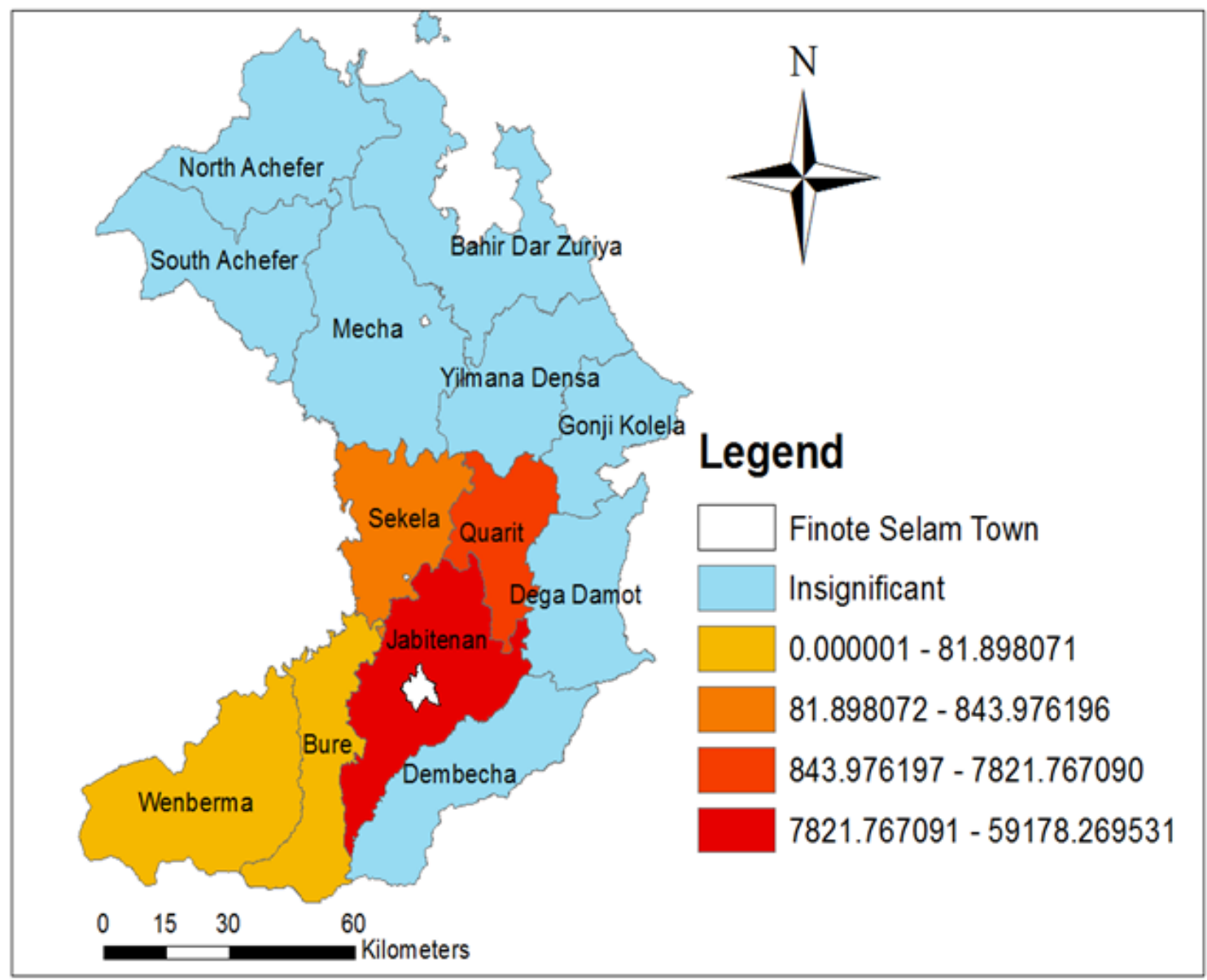

\section{Figure 5}

Spatial distribution of significant-high rate malaria clusters at a district level in West Gojjam, 1 July 2013-30 June 2018. Color identification of the clusters was ordered based on the value of likelihood ratio test statistic. 